\title{
Incomplete ECG Measurements Due to Truncation of QRS Complexes
}

National Cancer Institute

\section{Source}

National Cancer Institute. Incomplete ECG Measurements Due to Truncation of QRS

Complexes. NCI Thesaurus. Code C114179.

An electrocardiographic recording in which measurements (particularly of QRS

amplitudes) and/or interpretations are not performed because QRS complexes have been truncated. (CDISC) 